\title{
High-affinity $T$ helper epitope induces complementary helper and APC polarization, increased CTL, and protection against viral infection
}

\author{
Jeffrey D. Ahlers, ${ }^{1}$ Igor M. Belyakov, ${ }^{1}$ Elaine K. Thomas, ${ }^{2}$ and Jay A. Berzofsky ${ }^{1}$ \\ ${ }^{1}$ Molecular Immunogenetics and Vaccine Research Section, Metabolism Branch, National Cancer Institute, \\ National Institutes of Health, Bethesda, Maryland, USA \\ ${ }^{2}$ Immunex Corporation, Seattle, Washington, USA \\ Address correspondence to: Jay A. Berzofsky, Molecular Immunogenetics and Vaccine Research Section, \\ Metabolism Branch, National Cancer Institute, Building 10, Room 6B-12 (MSC\#1578), National Institutes of Health, \\ Bethesda, Maryland 20892-1578, USA. Phone: (301) 496-6874; Fax: (301) 480-0681; E-mail: berzofsk@helix.nih.gov.
}

Received for publication June 7, 2001, and accepted in revised form October 1, 2001.

\begin{abstract}
Natural viral proteins do not always make optimal vaccines. We have found that sequence modification to increase epitope affinity for class II MHC molecules (epitope enhancement) can improve immunogenicity. Here we show first that a higher-affinity helper epitope-enhanced HIV vaccine not only induces more cytotoxic T lymphocytes (CTLs), but also skews helper cells toward Th1 cytokine production and protects against HIV-1 recombinant vaccinia viral challenge. Furthermore, we elucidate a novel mechanism in which the higher-affinity vaccine induces dramatically more effective helper cells with a higher level of CD40L per helper cell and more positive cells, which in turn more effectively conditions dendritic cells (DCs) for CTL activation in a second culture. The improved helper cells also induce much greater IL-12 production by DCs, accounting for the reciprocal T helper polarization to Th1, and increase costimulatory molecule expression. Thus, increasing affinity for class II MHC results in a complementary interaction in which $T$ helper and antigen-presenting cells polarize each other, as well as increase CTL, and provide greater vaccine efficacy against viral infection.
\end{abstract}

J. Clin. Invest. 108:1677-1685 (2001). DOI:10.1172/JCI200113463.

\section{Introduction}

Viral proteins have not evolved to be optimal vaccines. We have demonstrated previously proof of principle for the approach of "epitope enhancement," whereby modifying the amino acid sequence of a Th epitope in the HIV-1 envelope to enhance binding to a class II MHC molecule can increase immunogenicity $(1,2)$. Attaching this modified Th epitope to a cytotoxic $\mathrm{T}$ lymphocyte (CTL) epitope enhanced CTL induction in vivo (2). Genetic mapping showed that the enhanced $\mathrm{CD} 8^{+} \mathrm{CTL}$ response was due to an improved $\mathrm{CD}^{+}{ }^{+}$cell response. We (3) and others (4-6) have shown similar epitope enhancement for peptides binding to class I MHC molecules. However, the molecular mechanisms by which increased affinity for class II MHC translates into increased efficacy remain unclear. Is the enhanced peptide simply active at lower concentrations? Is the difference only quantitative, or are there qualitative differences in the response? One might have expected a peptide with higher affinity for MHC to be more immunogenic, but not necessarily to induce a qualitatively different response.

Here we focus on the mechanism of epitope enhancement for class II MHC-restricted helper T cells in augmenting a CTL response. We and others have found an essential role for $\mathrm{CD}^{+} \mathrm{T}$ helper cells in induction of CTL responses to peptide vaccines (7-9). The mechanism of $\mathrm{CD}^{+}$cell help for CTL induction appears to involve an intermediate step requiring conditioning of the APC via CD40 ligation by CD40L (10-13), but the downstream effects of this ligation have not been determined. Although an upregulation of essential costimulatory molecules on the antigen-presenting cells (APCs) has been suggested as a possible mechanism of help for CTL induction, the identity of the specific costimulatory molecules involved and the mechanism enhancing $\mathrm{CD}^{+} \mathrm{CTL}$ activation remain unclear. Also, it was not known how the modified peptide increases help; we show here it acts by increasing CD40L expression. Furthermore, it was not known whether an increase in APC conditioning by augmentation of help would simply result in more effective antigen presentation or would qualitatively alter the APC to induce a more polarized response.

All of these questions are addressed in the current study. We find that modification of a Th cell epitope to increase binding to MHC causes quantitatively more $\mathrm{T}$ cells to undergo upregulation of CD40L and to express higher levels of CD40L per cell, which interacts with CD40 to condition the APC for CTL induction. The primary measurable effect of the improved APC conditioning by the higher-affinity peptide is upregulation of IL-12 production, along with some difference in B7-1 and B7-2 expression. Thus, unexpectedly, the APC becomes more polarizing toward a Th1 response. Furthermore, the conditioned APCs, removed from the Th 
cell, are more potent stimulators of CTL in vitro. Thus, epitope enhancement to increase peptide affinity for MHC class II results in a qualitative as well as quantitative change in help, through complementary interactions in which increased CD40L on the helper cells induces more polarizing APCs that act back on the T helper cell to produce a Th1-polarized response, as well as higher levels of CTL and protection against viral infection. Corollaries are that one mechanism to polarize APCs is to increase the stimulation through CD40 by CD40L and that peptide affinity for class II MHC can be used to mediate this effect for more effective vaccines.

\section{Methods}

Animals. BALB.A10 and A.AL mice were bred in our animal facility (BioCon Inc., Rockville, Maryland, USA) and used between 6 and 10 weeks of age. Both strains possess the class II molecule $\mathrm{E}^{\mathrm{k}}$, which bind Th peptides T1 and T1(A) and also express the class I $\mathrm{D}^{\mathrm{d}}$ molecule, which presents the HIV-1 CTL epitope P18IIIB.

Bone marrow-derived dendritic cells. Dendritic cells (DCs) were derived from bone marrow cultures according to the procedure used previously (14), with slight modification. Bone marrow cells were plated in Nunc flasks at $2 \times 10^{6}$ per $100 \mathrm{~mm} / 10 \mathrm{ml}$ in complete medium (2) plus GM-CSF (30 ng/ml; Peprotech Inc., Rocky Hill, New Jersey, USA) \pm IL-4 (200 U/ml; Life Technologies Inc., Grand Island, New York, USA). Day 7-9 cells were depleted of B cells, granulocytes, $T$ cells, and NK cells using an excess of anti-B220 coated (Dynal Inc., Lake Success, New York, USA) and biotinylated anti-CD3, R6B, and pan NK(DX5)-streptavidin-coated magnetic beads. Buoyant cells after centrifugation on Lympholyte $\mathrm{M}$ (CedarLane Laboratories Ltd., Hornsby, Ontario, Canada) were about $80 \% \mathrm{CD} 11 \mathrm{c}^{+}$. In some experiments CD11 $\mathrm{c}^{+}$cells were positively selected using magnetic beads (Miltenyi Biotech, Auburn, California, USA).

Antigen. Multideterminant cluster peptides (15) containing overlapping T helper epitopes were colinearly synthesized with P18IIIB, an immunodominant CTL epitope in mice (16) to form a synthetic peptide vaccine construct (17). The constructs used for immunization were PCLUS 3-18IIIB, KQIINMWQEVGKAMYAPPISGQIRRIQRGPGRAFVTIG, composed of HIV-1 IIIB envelope amino acid residues 421-444 according to Los Alamos data base numbering (18), linked to P18IIIB shown in bold (amino acid residues 308-322), and the corresponding construct with an epitope-enhanced helper portion, PCLUS 3(A)-18IIIB, KQIINMWQAVGKAMYAPPISGQIRRIQRGPGRAFVTIGK. The helper peptide PCLUS 3 contains the native Th helper epitope, T1 KQIINMWQEVGKAMYA, whereas PCLUS 3(A) contains the modified Th epitope, T1A, KQIINMWQAVGKAMYA, created by replacing Glu with Ala. T1(A) has higher affinity for the I-Ek (1). Peptides (synthesized by Multiple Peptide Systems, San Diego, California, USA) were single peaks as seen by HPLC and by mass spectrometry.
Viruses. Recombinant vaccinia virus vPE16 expressing HIV-IIIB gp 160 was a kind gift of Bernard Moss and Patricia Earl, National Institute of Allergy and Infectious Diseases, NIH (19). Virus was injected intraperitoneally into female mice in protection experiments, and 5 days later, ovaries were homogenized and sonicated and virus titered by plaque assay on BSC-1 cells (20).

Generation of Thelper line. Spleen cells from BALB.A10 congenic mice $\left(\mathrm{H}-2 \mathrm{E}^{\mathrm{k}}\right)$ immunized twice subcutaneously with PCLUS 3-18IIIB in Montanide ISA-51 (a kind gift of Jean-Pierre Planchot, Seppic Inc., Fairfield, New Jersey, USA) were stimulated twice with T1(A) under Th1-polarizing conditions: IL-12 $(10 \mathrm{ng} / \mathrm{ml})$ and anti-IL-4 $(10 \mu \mathrm{g} / \mathrm{ml})$. CD4 ${ }^{+}$cells were positively selected with anti-CD4 beads (Dynal Inc.) and cloned by limiting dilution. Th 1 clones expressing IL-2 and IFN- $\gamma$, but not IL-4, were selected. Proliferation was assayed by $\left[{ }^{3} \mathrm{H}\right]$ thymidine incorporation of $3 \times 10^{4}$ to $5 \times 10^{4} \mathrm{Th}$ cells stimulated with T1(A) and $5 \times 10^{5}$ irradiated syngeneic spleen cells in 96-well plates for 96 hours.

Cytotoxicity assay. A.AL (I-Ek, $\mathrm{D}^{\mathrm{d}}$ ) and BALB.A10 (I-Ek, $\left.D^{d}\right)$ mice were immunized subcutaneously with vaccines (20 nmol) in Montanide ISA-51. Spleen cells $\left(10^{7}\right)$ were stimulated with $10^{7}$ peptide-pulsed $(0.5 \mu \mathrm{M}$ P18IIIB for 1.5 hours) naive irradiated ( $30 \mathrm{~Gy}$ ) spleen cells in 12-well plates (Corning Costar Corp., Acton, Massachusetts, USA). Twelve hours later Rat T Stim (Collaborative Biomedical Research, Bedford, Massachusetts, USA) was added as a source of IL-2 (final concentration 7.5\%). Nonadherent cells 4 days later were centrifuged over mouse Lympholyte M (CedarLane Laboratories Ltd.), replated with $2.5 \mathrm{U} / \mathrm{ml}$ rmIL-2, and assayed on day 7-8 in triplicate in 96-well round-bottom plates on 5,000 target cells, either $18 \mathrm{Neo}\left(3 \mathrm{~T} 3\right.$ fibroblasts neo ${ }^{\mathrm{R}}$ ) or P815 P18IIIB pulsed or unpulsed in a 5-hour assay. Spontaneous lysis was less than or equal to $10 \%$. CTL data were normally distributed based on the Shapiro-Wilk test, and means are plotted as arithmetic means plus or minus SEM. Error bars smaller than the symbols could not be shown. Lysis without peptide was less than $10 \%$, except where indicated.

$R T-P C R$. Total RNA was isolated from $\mathrm{CD}^{+} \mathrm{T}$ cells 12-20 hours after in vitro restimulation, mRNA was reversed transcribed as described (21), and PCR was performed under nonsaturating conditions using primers described previously (21) for the housekeeping gene HPRT and murine cytokines and controls: IL-2, IL-4, IL-10, and IFN- $\gamma$ (CLONTECH Laboratories, Palo Alto, California, USA).

ELISA. Duplicate samples of $100 \mu \mathrm{l}$ of 48 - to 72 -hour culture supernatants from $5 \times 10^{5} \mathrm{CD}^{+}$cells stimulated in vitro with peptide and $5 \times 10^{5} \mathrm{DCs}$, were tested in an IFN- $\gamma$ ELISA assay (Life Technologies Inc.). Fortyeight-hour culture supernatants were tested for IL-12 with an IL-12 (p40 and p70) set (OPTEIA; PharMingen, San Diego, California, USA).

$D C$ conditioning cultures. Peptide T1, or T1(A), or no peptide was added to $5 \times 10^{5}$ DCs and $5 \times 10^{4}$ peptidespecific $\mathrm{CD}^{+} \mathrm{Th}$ cells in 12 -well plates and incubated 
for 15-18 hours. T cell-DC conjugates were disrupted by washing with HBSS with 1\% BSA, 25 mM HEPES, and $15 \mathrm{mM}$ EDTA. CD4 ${ }^{+} \mathrm{T}$ cells were removed with an excess of anti-CD4-coated magnetic beads (Dynal Inc.), and the remaining conditioned APCs were pulsed with the CTL epitope P18IIIB $(0.5 \mu \mathrm{M})$ for 1 hour. Pulsed DCs were irradiated and replated at $5 \times 10^{5}$ per well in 12 -well plates with $5 \times 10^{6} \mathrm{CD}^{+}$ cells negatively selected from spleen cells from animals primed in vivo more than 1 month earlier with PCLUS 3-18IIIB. Control DCs were incubated with GM-CSF only, or Th cells without peptide, or with murine trimeric CD $40 \mathrm{~L}$ as a positive control $(3 \mu \mathrm{g} / \mathrm{ml}$; Immunex Corp., Seattle, Washington, USA). In blocking experiments, $\mathrm{Ab}$ 's were added to $\mathrm{DC}-\mathrm{CD}^{+}{ }^{+}$cell cultures at the initiation of culture and on day 3.5. Blocking mouse Ab's used at $10-15 \mu \mathrm{g} / \mathrm{ml}$ - anti-B7-1 (16-10A1), anti-B7-2 (GL1), anti-ICAM (3E2), antiCD44H (TM-1), anti-IL-12 (C17-8) - were obtained as no azide/low endotoxin preparations from PharMingen or dialyzed overnight at $4^{\circ} \mathrm{C}$ against PBS; murine anti-41BBL was a kind gift from Immunex Corp.

FACS analysis. T1-Th1 cells $\left(3 \times 10^{4}\right)$ were incubated in 96-well round-bottom plates with $3 \times 10^{5} \mathrm{DCs}$ or purified naive splenic B cells $\left(\mathrm{B} 220^{+}\right)$and T1 or T1(A) peptide. Phycoerythrin-conjugated (PE-conjugated) rat anti-mouse CD40L was added initially and again 20 hours later. FITC-labeled rat anti-mouse $\mathrm{CD}^{+}$cells were examined for CD40L expression by FACScan (Becton Dickinson Immunocytometry Systems, San Jose, California, USA). Cultures of DCs unconditioned or conditioned by $\mathrm{CD} 4^{+} \mathrm{T} 1$-specific $\mathrm{Th}$ cell clones or CD40L (Immunex Corp.) for 24-36 hours were stained with PE-CD $11 \mathrm{c}^{+}$and FITC class I $\left(\mathrm{D}^{\mathrm{d}}\right)$, FITC anti-class II (E $\left.{ }^{\mathrm{k}}, 14.4 .4\right)$, FITC B7-1, B7-2, ICAM, CD44H, CD11b, CCR5, and CD40 (PharMingen), and anti-41BBL (Immunex Corp.). PE-conjugated $\mathrm{H}-2 \mathrm{D}^{\mathrm{d}} / \mathrm{I}-10$ peptide tetramer staining was performed as described previously (22) in the presence of Fc block (anti-CD16/CD32).

\section{Figure 1}

Epitope enhancement of Th epitope in Th-CTL peptide vaccine construct elicits enhanced CTL response. (a) BALB/c mice were immunized subcutaneously with $20 \mathrm{nmol}$ native vaccine construct PCLUS 3-18IIIB or construct with the modified helper epitope PCLUS 3(A)$18 \mathrm{IIIB}$. Spleen cells were restimulated in vitro 14 days later with irradiated P18IIIB-pulsed syngeneic spleen cells, and 7 days later tested in a ${ }^{51} \mathrm{Cr}$-release assay on P815 targets pulsed with $0.5 \mu \mathrm{M}$ P18IIIB. Open symbols represent nonspecific lysis of target cells without peptide. Curves are significantly different at $\alpha=0.001$ by Tukey multiple comparison test. (b) Draining lymph node cells from immunized animals were stimulated 4 days with P18IIIB and stained with anti-CD8 and $\mathrm{P} 18-110 / \mathrm{H}-2 \mathrm{D}^{d}$ tetramer. (c) Draining lymph node cells from immunized animals were tested immediately ex vivo without in vitro stimulation 3 days after immunization, in a 5-hour CTL assay. (d) Animals were challenged intraperitoneally with different doses of vPE16 14 days after two immunizations (primary immunization subcutaneously and boost intraperitoneally 14 days later). Four days after challenge, viral titers in homogenized ovary tissue were determined. Each column represents the mean of three mice.
Statistical analysis. Means were plotted as geometric or arithmetic based on the distribution of data determined by the Shapiro-Wilk test. $\Delta$ means represent the experimental minus no-antigen control mean of triplicate samples. A two-way ANOVA was applied to CTL data, thereby averaging the respective values for each curve over all the effector/target $(\mathrm{E} / \mathrm{T})$ ratios. Means were compared using Tukey's multiple comparison test as described (23), and significance was determined at $\alpha=0.05$. IFN- $\gamma$ and IL-12 ELISA data were treated similarly or by the Student $t$ test at $P=0.01$.

\section{Results}

Modification of Th epitope in Th-CTL vaccine construct elicits enhanced $C D 8^{+} C T L$ response. To determine the effect on the CTL response of improving the helper epitope without altering the CTL epitope, we immunized BALB.A10 mice with PCLUS 3-18IIIB and the construct containing the modified helper epitope, PCLUS 3(A)-18IIIB, and measured CTL responses after in vitro stimulation. Immunization with PCLUS 3(A)-18IIIB resulted in greater CTL induction with an eightfold horizontal shift in $\mathrm{E} / \mathrm{T}$ ratio after a single immunization (Figure 1a). This
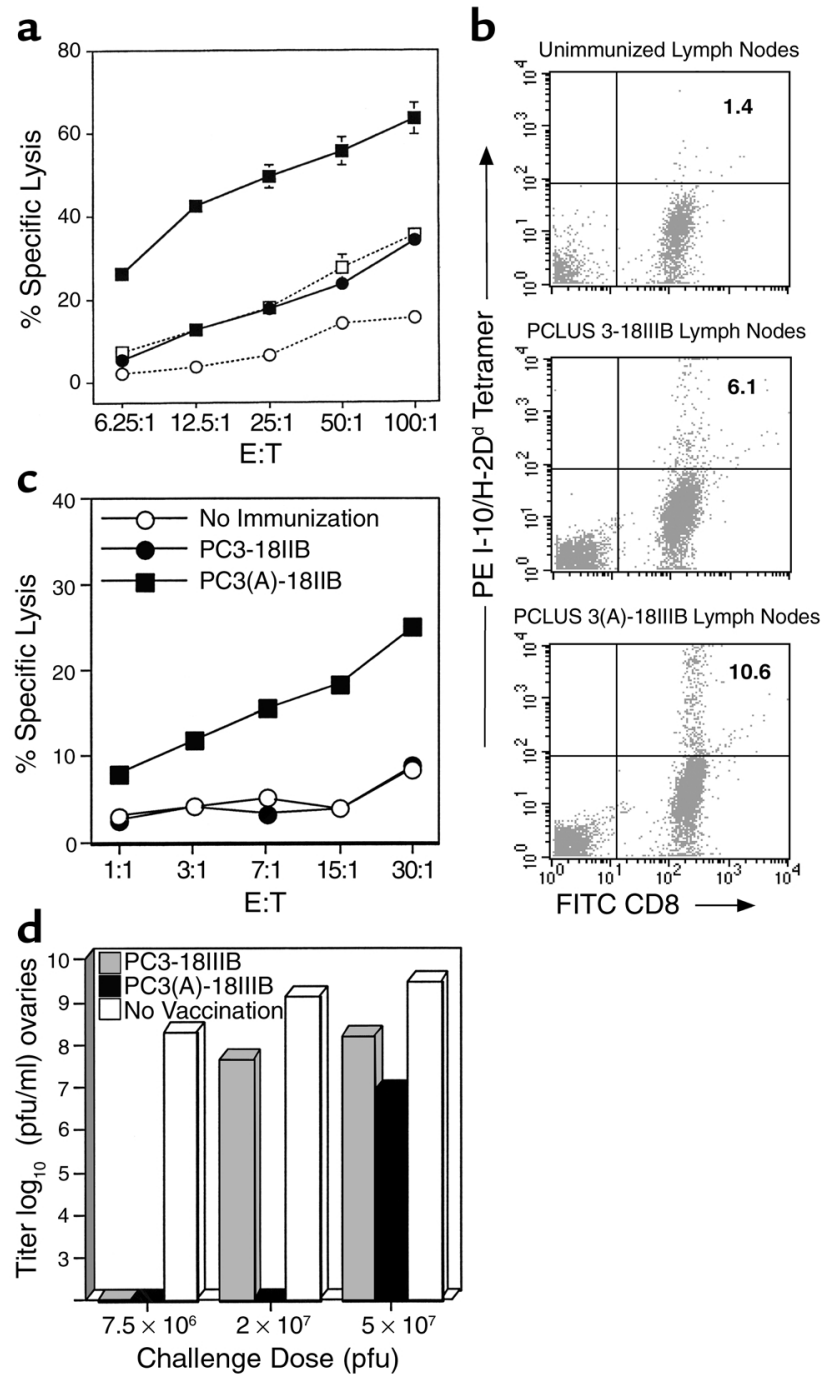


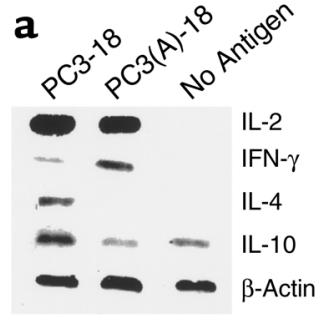

b

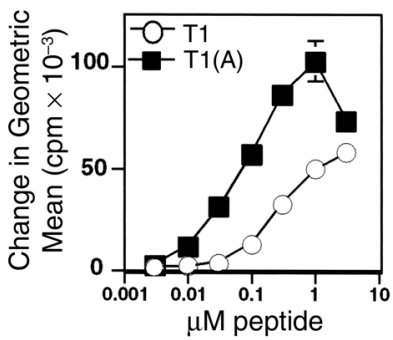

c

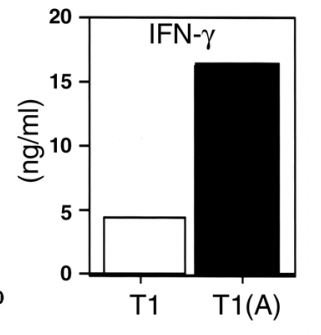

Figure 2

Higher-affinity binding peptide T1(A) skews T cells toward a Th1 response. (a) Inguinal and periaortic lymph node cells were harvested from animals immunized with PCLUS 3-18IIIB (PC3-18) or PCLUS 3(A)-18IIIB [PC3(A)-18] and stimulated overnight with the immunizing peptide at $3 \mu \mathrm{M}$. Total RNA was isolated from positively selected $C D 4^{+} T$ cells 20 hours after in vitro stimulation and semiquantitative RT-PCR performed under nonsaturating conditions to compare relative cytokine mRNA levels. These results were repeated with similar results in two additional experiments. (b) Stimulation of a $\mathrm{T} 1 \mathrm{CD} 4^{+} \mathrm{T}$ cell line with peptide $\mathrm{T} 1$ (A) elicited greater proliferation than when stimulated with T1. A similar level of proliferation was obtained at a 30 -fold lower concentration of peptide $\mathrm{T} 1(\mathrm{~A})$; compare proliferation at $0.1 \mu \mathrm{M} \mathrm{T} 1(\mathrm{~A})$ versus $3 \mu \mathrm{M} \mathrm{T} 1$. (c) IFN- $\gamma$ production over 48 hours as measured by ELISA from a T1-specific CD4 ${ }^{+} \mathrm{T}$ cell clone stimulated with peptide $\mathrm{T} 1$ or $\mathrm{T} 1(\mathrm{~A})$.

tion for induction of $\mathrm{CD} 8^{+} \mathrm{CTL}$ and to determine if the difference was purely quantitative or also qualitative, we measured cytokine mRNA in $\mathrm{CD}^{+}$cells from draining lymph nodes 3 days after immunization. Unexpectedly, a clear difference in cytokines was observed. The native Th epitope, T1, polarized CD4 ${ }^{+}$cells toward a Th2 phenotype as early as day 3 , whereas the construct containing the higher-affinity binding Th peptide T1(A) skewed the response toward Th1 (Figure 2a). In three separate experiments, IFN- $\gamma$ mRNA was at least 2.5-fold greater in animals immunized with the enhanced construct. In addition, IL-4 expression was either absent or decreased approximately threefold in $\mathrm{CD} 4^{+}$cells immunized with T1(A), and IL-10 expression was also reduced (Figure 2a). In contrast, we

increase in CTL is confirmed by a doubling of the specific $\mathrm{CD}^{+}$cells binding P18-I10/H-2 ${ }^{d}$ tetramers in draining lymph nodes after 4 days of stimulation with P18 peptide, from $4.7 \%$ to $9.2 \%$ after immunizing with PCLUS 3-18IIIB versus PCLUS 3(A)-18IIIB, respectively, with nonspecific background $1.4 \%$ in unimmunized mice subtracted (Figure 1b).

In a separate experiment, when the CTL activity was measured ex vivo without restimulation using draining lymph node cells 3 days after immunization, a significant CTL response was already evident in animals immunized with PCLUS 3(A)-18IIIB, but not in those immunized with PCLUS 3-18IIIB (Figure 1c). Thus, the modified Th epitope with higher affinity for MHC class II resulted in enhanced help for class I-restricted CTL induction as determined by greater numbers and activity and induction of $\mathrm{CD}^{+} \mathrm{CTL}$ without in vitro stimulation. Thus, help was indeed limiting for CTL induction.

To determine if this enhanced CTL response was more protective in vivo, we challenged immunized animals intraperitoneally with increasing doses of virus. We have found this model to be strictly dependent on the presence of activated CD8 ${ }^{+} \mathrm{CTL}$ at the site of viral challenge. Complete protection was achieved with both vaccine constructs at low-dose viral challenge; howev$\mathrm{er}$, at the intermediate dose complete protection was seen only in animals immunized with the enhanced (T1A)-vaccine construct (Figure 1d). Furthermore, at the highest challenge dose, virus was still inhibited by greater than $99 \%$ (100-fold) compared with 95\% (20fold) with the unmodified vaccine. Thus, greater induction of $\mathrm{CD}^{+} \mathrm{CTL}$ by immunization with the modified helper epitope-CTL vaccine construct correlated with improved protection from viral challenge.

Epitope modification polarizes the Th response to Th1 phenotype. To determine the nature of enhanced helper func- did not observe a significant difference in proliferation or IL-2 expression (Figure 2a and data not shown).

The difference between the two peptides in vivo was also reflected in vitro in an established T1-specific Th1 clone for which the proliferative dose-response curve was shifted 30 -fold, and IFN- $\gamma$ production significantly increased when stimulated with the higher-affinity peptide T1(A) (Figure 2, b and c). Because we have observed recently that selection of IFN- $\gamma$-producing $\mathrm{CD}^{+}$cells require a higher threshold of signaling through the TCR (24), the increased strength of signal delivered by the higher-affinity peptide could, in part, explain these results. We hypothesized that the higheraffinity peptide T1(A) may produce a stronger CD40L signal to activate DCs to produce IL-12, producing a more polarizing APC. Subsequent experiments were designed to test this hypothesis.

Differential upregulation of CD4OL on $\mathrm{CD}^{+} \mathrm{T}$ cells stimulated with high-affinity peptide T1(A). To examine the mechanism of improved help for CTL, we stimulated a $\mathrm{CD} 4^{+} \mathrm{Th} 1$ cell clone specific for peptide $\mathrm{T} 1$ with either $\mathrm{T} 1$ or $\mathrm{T} 1(\mathrm{~A})$ and examined expression of CD40L over a range of 2 logs antigen dose (Figure 3a). At the highest dose tested $(5 \mu \mathrm{M})$, all $\mathrm{CD}^{+}$cells expressed CD40L regardless of stimulating peptide used; however, levels of expression were markedly higher on $T$ cells stimulated with the higher-affinity peptide, T1(A). In addition, at decreasing doses of antigen, more $\mathrm{CD} 4^{+}$cells were activated to express CD40L (at $0.05 \mu \mathrm{M}$ peptide $68 \%$ versus $26 \%$ ), and these expressed more CD $40 \mathrm{~L}$ per cell when stimulated with $\mathrm{T} 1(\mathrm{~A})$. The dose for inducing CD40L expression was reduced by greater than or equal to $1 \log$ when T1(A) was used. Furthermore, this increase in CD40L expression was found when using either DCs (Figure 3a) or purified splenic B cells (Figure $3 b$ ) as APCs. These results were highly reproducible 

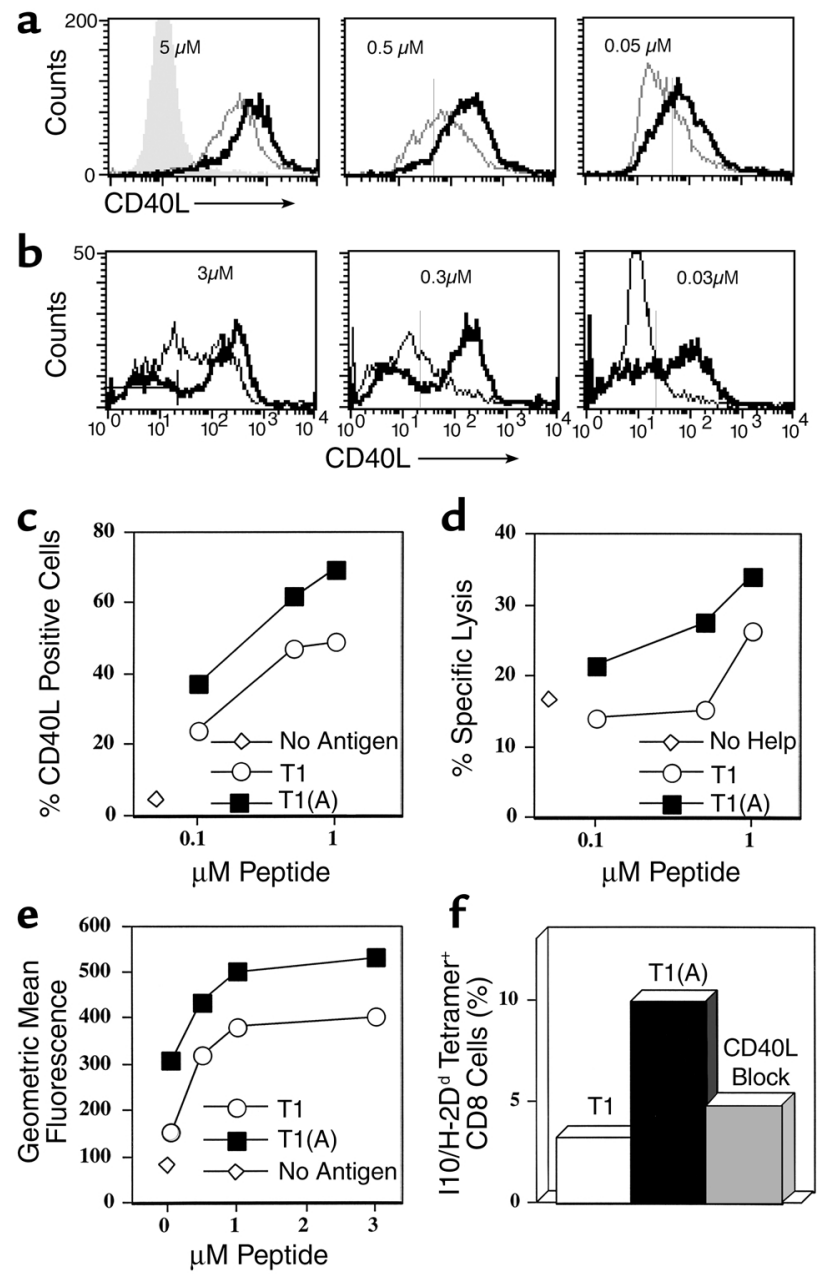

in four additional experiments. Thus, we show unexpectedly that increasing the affinity of the helper epitope for the class II MHC molecule results not only in increased numbers of activated helper $\mathrm{T}$ cells, but also in qualitatively different help, with more CD40L per cell and polarized to Th1 cytokines.

Enhanced helper function through conditioning and polarizing of APCs. To determine if enhanced T helper CD40L expression was responsible for the enhanced CTL induction in vivo, we did a two-step mixing experiment in which peptide-specific $\mathrm{CD} 4^{+} \mathrm{Th} 1$ cells were stimulated overnight with different concentrations of peptide and DCs, and then the resulting DCs were used to stimulate CTL. The first stage, CD40L expression on gated $\mathrm{CD} 4^{+}$cells 20 hours after stimulation with $\mathrm{T} 1$ and T1(A), is shown in Figure 3c. Stimulation with the modified Th epitope T1(A) elicited more total CD40L ${ }^{+}$ $\mathrm{T}$ cells at all antigen concentrations (e.g., $72 \%$ vs. $50 \%$ at $1 \mu \mathrm{M}$ peptide). There was a clear dose-response effect. Parallel cultures of the same cell preparation were then depleted of $\mathrm{CD} 4^{+} \mathrm{T}$ cells to recover the DCs, which were then pulsed with the CTL epitope peptide P18IIIB, irradiated, and mixed with $\mathrm{CD}^{+} \mathrm{T}$ cells enriched from spleens of animals immunized with PCLUS 3-18IIIB. The level of CTL activity induced by these conditioned

\section{Figure 3}

Qualitative and quantitative upregulation of CD40L on CD4 ${ }^{+} T$ cells stimulated with $\mathrm{T} 1(\mathrm{~A})$ and concordant increased activation of DCs to stimulate CTL. (a) CD40L expression on T1-specific CD4 ${ }^{+} \mathrm{T}$ cells incubated overnight with different concentrations of T1 (dashed line) or T1 (A) (solid line) and DCs, or without peptide (shaded area). (b) CD40L expression on gated CD4+ ${ }^{+} 1$-specific $T$ cells stimulated 36 hours with indicated concentrations of $\mathrm{T} 1$ or $\mathrm{T} 1(\mathrm{~A})$ peptide and naïve A.AL splenic B cells (B220+ selected). The bar in the left panel indicates background CD40L without peptide. (c) Percentage $\mathrm{CD}^{+} /$ CD40L ${ }^{+}$cells plotted versus peptide concentration for Th1 $\left(5 \times 10^{4}\right.$ $\mathrm{CD}^{+}$) cells incubated 24 hours with $5 \times 10^{5}$ BALB.A10 DCs and T1 or T1(A) peptide, or no peptide (open diamond). (d) $5 \times 10^{6} \mathrm{DCs}$ of the same preparation as in $\mathbf{c}$ were incubated with different concentrations of peptide and $5 \times 10^{5}$ of the same CD4 $4^{+}$cells, but in 12 well plates. $\mathrm{CD} 4^{+}$cells were depleted by magnetic beads 24 hours later. Remaining DCs were pulsed with $0.5 \mu \mathrm{M}$ CTL epitope P18IIIB, irradiated, and $5 \times 10^{6}$ added to $5 \times 10^{6}$ purified $C D 8^{+}$cells from spleens of 3 mice immunized 8 weeks earlier with PCLUS3-18IIIB, and rmIL-2 $(10 \mathrm{U} / \mathrm{ml})$ was added on day 0 . Specific lysis 7 days later on P18IIIB-pulsed P815 targets at a 50:1 E:T ratio is plotted versus Th peptide concentration used in conditioning cultures, or without Th peptide (open diamonds). Similar results were obtained in 2 other experiments. (e) Level of CD40L expression on the Th cells as a function of peptide concentration, using less rested Th cells which were 93\% CD40L $\mathrm{L}^{+}$even without stimulation. (f) P18-110/H-2Dd tetramer staining of $\mathrm{CD}^{+}$cells stimulated with $1 \mu \mathrm{M}$ P18-pulsed DC conditioned with Th cells from the same experiment as in (e) (less control $3.3 \%$ with unconditioned DCs).

DCs (Figure 3d) closely paralleled the level of CD40L expression induced on the Th cells used to condition the DCs (Figure 3c). In a separate experiment using less-rested Th cells already 93\% CD40L positive, the level of CD40L per cell was increased significantly more by T1(A) than by T1 (Figure 3e), and the resulting DCs induced twice the percentage of $\mathrm{CD} 8{ }^{+}$cells that were P18-I10/H-2Dd tetramer-positive (Figure 3f). Furthermore, blocking CD40L on the Th cell stimulated with peptide T1(A) abrogated IL-12 production by DCs (Figure 4 ) and reduced the CTL response (Figure $3 f$ and see Figure 6a), formally establishing a link between the enhanced helper function and CTL induction. Thus we conclude that the epitope-enhanced helper peptide is more effective at inducing CTL because it better upregulates CD40L on the Th cell, which then more effectively conditions APCs to better activate CTL after the Th cells have been removed.

CD40L expression on $\mathrm{CD} 4^{+}$cells stimulated with higheraffinity binding peptide T1 A) correlates with enhanced IL-12 production by DCs, resulting in polarizing DCs. To determine the molecular differences in the DCs that made those conditioned by T1(A) more effective at inducing CTLs, we examined cytokine production and surface expression of costimulatory molecules. T1-specific 


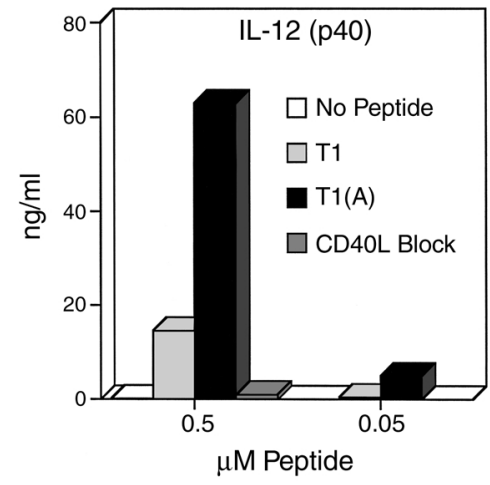

Figure 4

Induction of IL-12 production by DCs is dramatically increased by Th cells stimulated with higher-affinity peptide and is blocked by antiCD40L. Triplicate cultures of $5 \times 10^{4} \mathrm{~T} 1$-specific CD4 ${ }^{+} \mathrm{Th}$ cell clone were stimulated with $5 \times 10^{5} \mathrm{DCs}$, and 50 hours later culture supernatants were assayed for IL-12 by ELISA. These results were highly reproducible in three separate experiments.

$\mathrm{CD}^{+}$Th cells were stimulated in vitro with DCs and either $\mathrm{T} 1$ or $\mathrm{T} 1(\mathrm{~A})$ and $\mathrm{IL}-12$ production determined 48 hours later by ELISA for IL-12 (p40 subunit; with similar results for IL-12 p70 heterodimer, not shown). IL-12 production was increased more than threefold in cells stimulated with $\mathrm{T} 1(\mathrm{~A})$ compared with $\mathrm{T} 1$ at the optimum proliferative dose $(0.5 \mu \mathrm{M}$ peptide) (Figure 4$)$ and significantly increased at a log lower dose ( $0.05 \mu \mathrm{M}$ peptide). This effect was completely blocked by the addition of anti$\mathrm{CD} 40 \mathrm{~L} \mathrm{Ab}$. Therefore, the induction of IL-12 production by DCs is dependent upon CD40L-CD40 interaction, and epitope enhancement leads to reciprocal cross talk between the T cell and DC, increasing $\mathrm{CD} 40 \mathrm{~L}$ expression on the $\mathrm{CD} 4^{+}$Th cell and IL-12 production by the DC, thus skewing Th cell phenotype toward Th1. A further implication is that one mechanism to generate polarizing APCs skewed toward Th1 induction is to increase the helper signal with a higher-affinity peptide.

Differential upregulation of costimulation on DCs by higher-affinity Th epitope T1(A). We also wanted to determine if enhanced CD40L expression contributed to an upregulation of any costimulatory molecules on the APCs. Bone marrow-derived DCs were incubated with peptide $\mathrm{T} 1$ or T1(A) and the T1-specific Th clone for 24 hours. Cells were gated on class II or $\mathrm{CD} 11 \mathrm{c}^{+}$and examined for costimulatory molecules, B7-1, B7-2, ICAM, CD44H, 41BBL, CCR5, and CD40. In contrast to class II expression, B7-1, B7-2, and CD40, were

Figure 5 upregulated in cells conditioned with Th and either peptide, but B7-1 expression was greater with the higher-affinity peptide T1(A) (Figure 5a). In a subsequent experiment (Figure $5 \mathrm{~b}$ ), when we gated on $\mathrm{CD} 11 \mathrm{c}^{+}$ cells, we found a marked increase in the level and numbers of cells expressing both B7-1 and B7-2 in DCs conditioned with the Th stimulated with T1(A). Although we always observed an upregulation of both ICAM and CD44H in cultures incubated with peptide compared with those without, increases were similar with either peptide, as was true for 41BBL or CCR5 (data not shown).

We performed blocking experiments to determine whether these molecules were necessary for the enhanced CTL induction. DCs conditioned overnight with T1(A) and T1-specific Th cells were depleted of $\mathrm{CD}^{+}$cells, pulsed with $\mathrm{P} 18 \mathrm{IIIB}$, and added to purified $\mathrm{CD}^{+}$cells from mice immunized with PC3-18IIIB. DCs conditioned with the T1(A) or CD40L elicited significantly higher levels of lysis than those conditioned with T1 or with Th-cells only, without peptide (Figure 6a). Furthermore, the enhanced CTL response could be blocked by the addition of anti-CD $40 \mathrm{~L}(10 \mu \mathrm{g} / \mathrm{ml})$ during the initiation of culture, down to the level found with DCs preincubated with Th cells only, without peptide. (The high background with DCs that were not
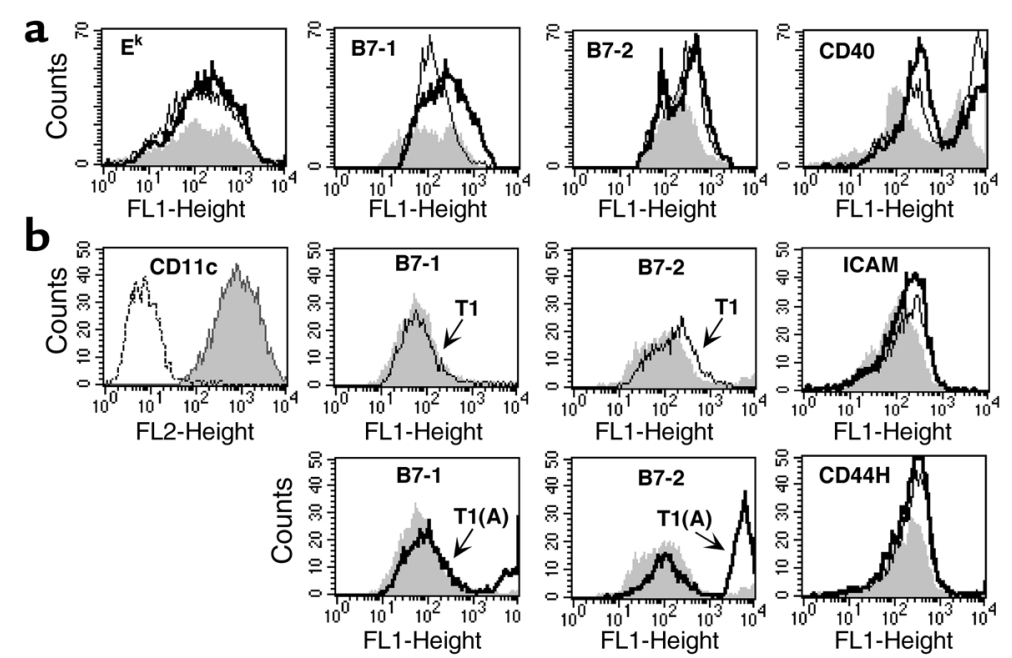

Differential expression of costimulatory molecules. Eight-day A.AL DC cultures $\left(5 \times 10^{5}\right.$ cells/well of 96 -well round-bottom plate) grown with GM-CSF $(10 \mathrm{ng} / \mathrm{ml})$ were conditioned for 20 hours by incubation with $5 \times 10^{4} \mathrm{CD}^{+}$Th cells and $0.5 \mu \mathrm{M}$ peptide $\mathrm{T} 1$ or $\mathrm{T} 1(\mathrm{~A})$. (a) Gated class $\mathrm{II}^{+}$cells were examined for expression of CD40 and costimulatory molecules B7-1 and B7-2 after stimulation with T1(A) (solid heavy line) compared with T1 (light line). DC cultures incubated with the Th cell without peptide are shown as the shaded area and are similar to cultures incubated without Th cells (not shown). (b) Eight-day BALB.A10 DCs were conditioned similarly for 36 hours. Gated CD1 $1 c^{+}$shown in the first panel as the shaded area compared with PE-isotype control (dashed line) were examined for costimulatory molecules B7-1, B7-2, ICAM, and $\mathrm{CD} 44 \mathrm{H}-\mathrm{DC}$ conditioned with Th cells stimulated with T1(A) (solid heavy line) versus T1 (light line). The shaded area represents DC cultures incubated with resting Th cells not stimulated with peptide. CD40 was maximally expressed under all culture conditions in this experiment. For B7-1 and 2, T1 and $\mathrm{T} 1(\mathrm{~A})$ are shown in separate panels for clarity. Similar results were observed in five additional experiments. 
a

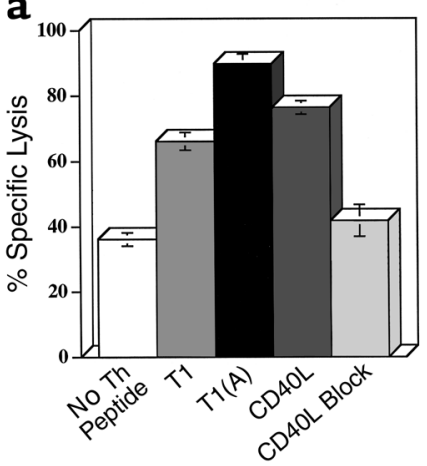

b

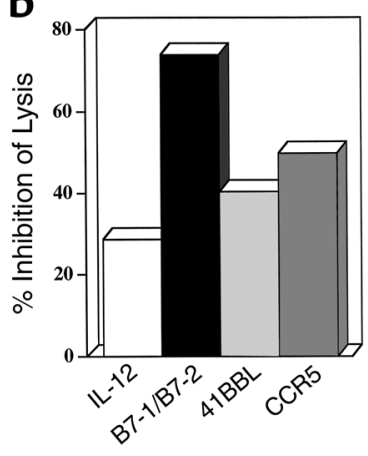

\section{Figure 6}

Blocking both $B 7-1$ and $B 7-2$ on DCs inhibits the CTL response. BALB.A10 DC cultures $\left(2.5 \times 10^{6}\right)$ conditioned for 24 hours with Th cells and peptide, T1, or T1 (A) $(0.5 \mu \mathrm{M})$, or no peptide, were depleted of $\mathrm{CD} 4^{+}$cells, pulsed for 1 hour with the CTL epitope P18IIIB $(0.5$ $\mu \mathrm{M})$, irradiated, and added to $5 \times 10^{6}$ negatively selected splenic $\mathrm{CD}^{+}$cells from BALB.A10 mice primed 1 month earlier subcutaneously with PCLUS 3-18IIIB (20 nmol). (a) DCs conditioned with Th cell and $T 1(A)$ elicited higher levels of CTL than DCs conditioned with either Th cells and T1 or no peptide, or overnight treatment with trimeric murine CD40L $(3 \mu \mathrm{g} / \mathrm{ml})$. Lytic activity was determined on day 7 in a 5 -hour ${ }^{51} \mathrm{Cr}$ assay on $0.5 \mu \mathrm{M}$ P18IIIB-pulsed P815 target cells $(E / T=10: 1)$. (b) DCs were conditioned with Th cells and T1 $(A)$ $(0.5 \mu \mathrm{M})$ for 24 hours and $\mathrm{CD}^{+}$cells removed. Cells were pulsed for 1.5 hours with $\mathrm{P} 18 \mathrm{II} \mathrm{B}$, irradiated, and used to stimulate immune CD8 ${ }^{+}$cells. Anti-B7-1/B7-2 (15 $\mu \mathrm{g} / \mathrm{ml}$ each), anti-IL-12 $(15 \mu \mathrm{g} / \mathrm{ml})$, anti-CCR5 $(10 \mu \mathrm{g} / \mathrm{ml})$, or anti-41BBL $(10 \mu \mathrm{g} / \mathrm{ml})$ were added to pulsed DCs at the initiation of CTL culture and on day 3 . Similar results were obtained in two additional experiments.

conditioned by $\mathrm{CD} 4^{+}$Th cells and peptide may be due to in vitro maturation of DCs). The addition of both anti-B7-1/B7-2 significantly decreased CTL induction (Figure 6b), where anti-IL-12, anti-41BBL, or anti-CCR5, partially reduced CTL. In six separate blocking experiments we could reproducibly inhibit CTL induction with the combination anti-B7-1/B7-2. We could not inhibit CTL with $\mathrm{Ab}$ to $\mathrm{B} 7-1$ or $\mathrm{B} 7-2$ alone, ICAM, CD44H (alone or in combination), or reproducibly inhibit CTL by greater than $40 \%$ with anti-IL12, anti-41BBL, or anti-CCR5 (Figure $6 \mathrm{~b}$ and data not shown). Thus, the major effect of the $\mathrm{CD}^{+} \mathrm{Th}$ cell in conditioning DCs for enhanced CTL induction occurs through CD40L-CD40 interaction and is dependent on upregulation of both B7-1 and B7-2. Upregulation of IL-12 production appears to be another major effect of conditioning, although blocking by anti-IL-12 was only partial. The increased IL-12 expression at least accounts for the DC's becoming more Th1 polarizing, but may be in sufficient excess for CTL induction that Ab blockade was incomplete. Thus, we conclude that the enhanced peptide with higher affinity for MHC class II acts by upregulating CD40L on the Thelper cell, which in turn acts through CD40 on the DC to upregulate both B7-1/B7-2 and IL-12 production, thus making a more potent DC for CTL induction and also a more polarizing one for Th1 induction (Figure 7).

\section{Discussion}

These experiments define a mechanism of enhanced CTL induction by modification of a $\mathrm{CD}^{+} \mathrm{T}$ helper cell epitope. We have found not only quantitative but also qualitative improvement in the immune response through epitope enhancement and have dissected each step of the process (Figure 7). The improved peptide increases $\mathrm{T}$ helper activation by augmenting CD40L expression, which in turn increases activation of DCs and alters the DCs qualitatively, making them more Th1 polarizing through increased IL-12 production. The conditioned DCs, through higher IL-12 and B7-1 and B7-2, more effectively activate CTL and induce better protection against viral infection, but also feed back on the helper $\mathrm{T}$ cells to polarize them more toward Th1 phenotype.

We have shown that $\mathrm{CD}^{+} \mathrm{T}$ cells are essential for induction of $\mathrm{CD}^{+} \mathrm{CTL}$ responses to peptide-based vaccines $(2,7,23)$, and other groups have shown a similar requirement for $\mathrm{CD}^{+}$help to many cell-based antigens $(9,13,25,26)$. However, it was not expected that CD4 help would be the limiting factor in improving a $\mathrm{CD}^{+}$ CTL response, such that altering the helper epitope affinity for class II MHC, without modifying the CTL epitope presented by class I MHC, would greatly augment CTL induction. It was also not known whether such increased help would increase protection against viral infection, as we now show. These findings have important implications for vaccine design.

The mechanism of $\mathrm{CD}^{+} \mathrm{T}$ cell help for CTL has been clearly shown to involve activation of immature DCs to a fully mature competent state through CD40L-CD40 interaction (10-12), but the effect of the CD40L on the conditioned DC that results in increased activity has not been determined. Upregulation of costimulatory molecules has been assumed, but not yet demonstrated. In this study, we find that increasing the affinity of a Th epitope for class II MHC more efficiently upregulates $\mathrm{CD} 40 \mathrm{~L}$ on $\mathrm{CD} 4^{+} \mathrm{Th} 1$

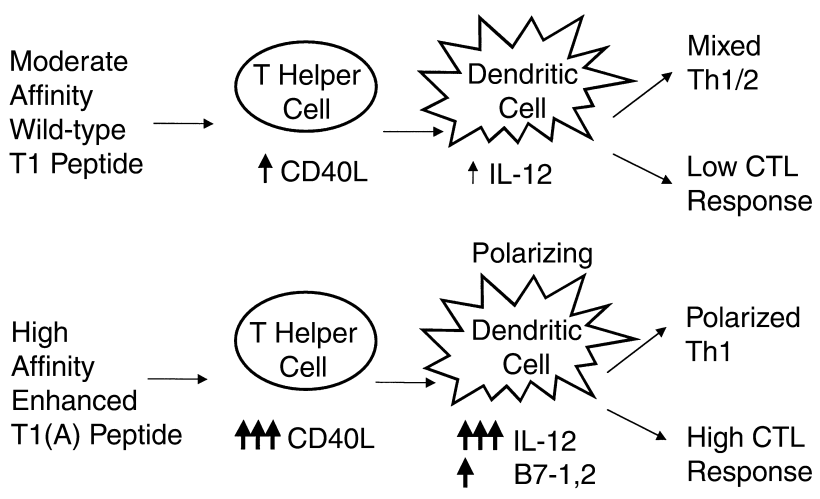

\section{Figure 7}

Mechanism of enhanced CTL induction and Th1 polarization by high-affinity peptide. Schematic representing the reciprocal interaction between the Th1 cell stimulated with either the native peptide $\mathrm{T} 1$ or the enhanced binding peptide $\mathrm{T} 1(\mathrm{~A})$ and the DC leading to induction of CTL, and to Th1 polarization. 
cells both quantitatively and qualitatively; i.e., both the number of $\mathrm{CD}^{4} 0 \mathrm{~L}^{+}$cells and the level per cell. Furthermore, the level of CD40L expression was shown to correlate directly to induction of IL-12 by DCs and blocking CD40L on the Th-cell abrogated IL-12 production. Although it was known that signals delivered to DCs through CD40 can induce IL-12 production $(27,28)$, it was unexpected that augmentation of help through increasing the density of CD40L on helper T cells would generate polarizing DCs that would in turn skew the helper response to Th1. This may be a general approach to produce such polarizing APCs. The biological significance is supported by a recent study showing that protective anti-tumor immunity required CD40-mediated activation of DCs (29).

Thus, a key effect of enhanced Th epitope affinity is increased IL-12 production. IL-12 acts in driving the selective expansion of Th1 cells $(23,30,31)$, enhances CTL proliferation (32), and may further upregulate costimulation (33). In fact, we observed that the higheraffinity peptide clearly skewed the Th phenotype to Th1. Thus, the strength of TCR signal determines levels of CD40L, which in turn determines IL-12 production by APCs and selection of a Th1 phenotype. In a two-step culture, the upregulation of CD40L on the Thelper cells in the first culture directly correlated with the ability of DCs conditioned in this culture to stimulate $\mathrm{CD} 8^{+} \mathrm{CTL}$ activity in a second culture. Blockade of increased CTL induction in the second culture by treatment of the helper cells in the first culture with anti-CD40L (Figure 6a) suggests that this correlation represents cause and effect. These results complement and help to explain the mechanism by which altered peptide ligands have been shown to skew Th phenotype in the opposite direction, toward Th2 (34-38). Thus, both complementary results strongly support the conclusion that strength of signal contributes to $\mathrm{T}$ helper polarization.

DCs activated by $\mathrm{CD}^{+}$help stimulated with the higher-affinity peptide T1(A) induced somewhat higher levels of expression of B7-1 and B7-2, but not other costimulatory molecules, ICAM, CD44H, or $41 \mathrm{BBL}$ (39), and the resulting increase in CTL was inhibited by blocking both B7-1 and B7-2. However, the increase in B7-1 and B7-2 was modest compared with that of IL-12 production. Conditioning thus appears to represent predominantly an increase in IL-12 production and secondarily an increase in B7-1 and B7-2. IL-15 production by activated DCs may also play a role in CTL proliferation $(40,41)$, which we can investigate as reagents for detecting murine IL-15 become available.

The ability of DCs to stimulate antigen-primed $\mathrm{CD}^{+}$ CTL independent of CD4 cells is dependent on the state of activation and maturation of DCs (42). Besides CD40L-CD40 interaction, inflammatory stimuli can also activate DCs for induction of CTL $(13,43,44)$. Thus, DCs may be activated and CTL elicited in both CD4-dependent and CD4-independent pathways (45). A recent study (46) showed that although $\mathrm{CD}^{+}{ }^{+} \mathrm{CTL}$ mediated resolution of acute lymphocyte choriomenin- gitis virus infection occurred in CD40L-deficient mice, chronic infection was not controlled, suggesting a need for CD40L-CD40 interaction and a polarized CD4 ${ }^{+} \mathrm{Th}$ cell response in resolving chronic viral infections.

In summary, while exploring the mechanisms behind the epitope enhancement effect, we discovered several novel basic mechanisms by which $T$ helper cells contribute to CTL induction and can generate polarizing APCs. Epitope enhancement to increase the affinity of a class II MHC binding peptide can dramatically enhance the efficacy of a Th-CTL peptide vaccine construct for CTL induction and protection against viral infection, both quantitatively and qualitatively. The enhanced peptide shifts the quality of the response toward Th1 by greater upregulation of $\mathrm{CD} 40 \mathrm{~L}$ on the helper $\mathrm{T}$ cells, which then more effectively condition and polarize the DCs by upregulation of B7-1 and B7-2 expression and IL-12 production, which improves CTL induction and skews the cytokine profile. Better understanding of the mechanism of epitope enhancement may lead to the rational design of more effective second-generation vaccines.

\section{Acknowledgments}

We thank Alfred Singer and Alan Sher for critical reading of the manuscript and helpful discussions; Patricia Earl and Bernard Moss for the recombinant vaccinia virus; Jean-Pierre Planchot for the Montanide ISA 51; and Immunex Corp. for the CD40L.

1. Boehncke, W.-H., et al. 1993. The importance of dominant negative effects of amino acids side chain substitution in peptide-MHC molecule interactions and $T$ cell recognition. J. Immunol. 150:331-341.

2. Ahlers, J.D., Takeshita, T., Pendleton, C.D., and Berzofsky, J.A. 1997. Enhanced immunogenicity of HIV-1 vaccine construct by modification of the native peptide sequence. Proc. Natl. Acad. Sci. USA. 94:10856-10861.

3. Sarobe, P., et al. 1998. Enhanced in vitro potency and in vivo immunogenicity of a CTL epitope from hepatitis $\mathrm{C}$ virus core protein following amino acid replacement at secondary HLA-A2.1 binding positions. J. Clin. Invest. 102:1239-1248.

4. Pogue, R.R., Eron, J., Frelinger, J.A., and Matsui, M. 1995. Amino-terminal alteration of the HLA-A*0201-restricted human immunodeficiency virus pol peptide increases complex stability and in vitro immunogenicity. Proc. Natl. Acad. Sci. USA. 92:8166-8170.

5. Parkhurst, M.R., et al. 1996. Improved induction of melanoma-reactive CTL with peptides from the melanoma antigen gp100 modified at HLAA*0201-binding residues. J. Immunol. 157:2539-2548.

6. Slansky, J.E., et al. 2000. Enhanced antigen-specific antitumor immunity with altered peptide ligands that stabilize the MHC-peptide-TCR complex. Immunity. 13:529-538.

7. Shirai, M., et al. 1994. Helper-CTL determinant linkage required for priming of anti-HIV CD8+CTL in vivo with peptide vaccine constructs. J. Immunol. 152:549-556.

8. Ahlers, J.D., et al. 1996. Candidate HIV type 1 multideterminant cluster peptide-P18MN vaccine constructs elicit type 1 helper T cells, cytotoxic $\mathrm{T}$ cells, and neutralizing antibody, all using the same adjuvant immunization. AIDS Res. Hum. Retroviruses. 12:259-272.

9. Bennett, S.R.M., Carbone, F.R., Karamalis, F., Miller, J.F.A.P., and Heath, W.R. 1997. Induction of a CD8+ cytotoxic T lymphocytes response by cross-priming requires cognate CD4+ T cell help. J. Exp. Med. 186:65-70.

10. Ridge, J.P., Di Rosa, F., and Matzinger, P. 1998. A conditioned dendritic cell can be a temporal bridge between a CD4+ T-helper and a T-killer cell. Nature. 393:474-478.

11. Bennett, S.R.M., et al. 1998. Help for cytotoxic-T-cell responses is mediated by CD40 signalling. Nature. 393:478-480.

12. Schoenberger, S.P., Toes, R.E.M., van der Voort, E.I.H., Offringa, R., and Melief, C.J.M. 1998. T-cell help for cytotoxic T lymphocytes is mediated by CD40-CD40L interactions. Nature. 393:480-483.

13. Schuurhuis, D.H., et al. 2000. Immature dendritic cells acquire CD8(+) 
cytotoxic $\mathrm{T}$ lymphocyte priming capacity upon activation by $\mathrm{T}$ helper cell-independent or -dependent stimuli. J. Exp. Med. 192:145-150.

14. Lutz, M.B., et al. 1999. An advanced culture method for generating large quantities of highly pure dendritic cells from mouse bone marrow. $J$. Immunol. Method.s 223:77-92.

15. Berzofsky, J.A., et al. 1991. Construction of peptides encompassing multideterminant clusters of HIV envelope to induce in vitro T-cell responses in mice and humans of multiple MHC types. J. Clin. Invest. 88:876-884.

16. Takahashi, H., et al. 1988. An immunodominant epitope of the HIV gp160 envelope glycoprotein recognized by class I MHC moleculerestricted murine cytotoxic T lymphocytes. Proc. Natl. Acad. Sci. USA 85:3105-3109.

17. Ahlers, J.D., et al. 1993. Construction of an HIV-1 peptide vaccine containing a multideterminant helper peptide linked to a V3 loop peptide 18 inducing strong neutralizing antibody responses in mice of multiple MHC haplotypes after two immunizations. J. Immunol. 150:5647-5665.

18. Kuiken, C., et al., 2000. HIV sequence compendium 2000. Los Alamos National Laboratory. Los Alamos, New Mexico, USA. 592. pp.

19. Earl, P.L., Hugin, A.W., and Moss, B. 1990. Removal of cryptic poxvirus transcription termination signals from the human immunodeficiency virus type 1 envelope gene enhances expression and immunogenicity of a recombinant vaccinia virus. J. Virol. 64:2448-2451.

20. Alexander-Miller, M.A., Leggatt, G.R., and Berzofsky, J.A. 1996. Selective expansion of high or low avidity cytotoxic T lymphocytes and efficacy for adoptive immunotherapy. Proc. Natl. Acad. Sci. USA. 93:4102-4107.

21. Ahlers, J.D., Belyakov, I.M., Matsui, S., and Berzofsky, J.A. 2001. Mechanisms of cytokine synergy essential for vaccine protection against viral challenge. Int. Immunol. 13:897-908.

22. Belyakov, I.M., et al. 2001. Activating CTL precursors to reveal CTL function without skewing the repertoire by in vitro expansion. Eur. $J$. Immunol. In press.

23. Ahlers, J.D., Dunlop, N., Alling, D.W., Nara, P.L., and Berzofsky, J.A. 1997. Cytokine-in-adjuvant steering of the immune response phenotype to HIV-1 vaccine constructs: GM-CSF and TNF-alpha synergize with IL-12 to enhance induction of CTL. J. Immunol. 158:3947-3958.

24. Ahlers, J.D., Belyakov, I.M., Matsui, S., and Berzofsky, J.A. 2001. Signals delivered through TCR instruct IL-12R expression: IL-12 and TNF $\alpha$ synergize for IL-12R expression at low antigen dose. Int. Immunol. 13:1433-1442

25. Takahashi, H., Nakagawa, Y., Yokomuro, K., and Berzofsky, J.A. 1993. Induction of CD8 + CTL by immunization with syngeneic irradiated HIV-1 envelope derived peptide-pulsed dendritic cells. Int. Immunol. 5:849-857.

26. Husmann, L.A., and Bevan, M.J. 1988. Cooperation between helper T cells and cytotoxic T lymphocyte precursors. Ann. NY Acad. Sci. 532:158-169.

27. Cella, M., et al. 1996. Ligation of CD40 on dendritic cells triggers production of high levels of interleukin-12 and enhances T cell stimulatory capacity: T-T help via APC activation. J. Exp. Med. 184:747-752.

28. Ding, L., and Shevach, E.M. 1998. Differential effects of CD28 engagement and IL-12 on T cell activation by altered peptide ligands. $J$. Immunol. 161:6614-6621.
29. Mackey, M.F., et al. 1998. Dendritic cells require maturation via CD40 to generate protective antitumor immunity. J. Immunol. 161:2094-2098.

30. Macatonia, S.E., Hsieh, C.-S., Murphy, K.M., and O'Garra, A. 1993. Dendritic cells and macrophages are required for Th1 development of CD4+ $\mathrm{T}$ cells from ab-TCR transgenic mice: IL-12 substitution for macrophages to stimulate IFN-g production is IFN-g-dependent. Int Immunol. 5:1119-1128.

31. Macatonia, S.E., et al. 1995. Dendritic cells produce IL-12 and direct the development of Th1 cells from naive CD4+ T cells. J. Immunol. 154:5071-5079

32. Trinchieri, G. 1998. Proinflammatory and immunoregulatory functions of interleukin-12. Intern. Rev. Immunol. 16:365-396.

33. Kelleher, P., and Knight, S.C. 1998. IL-12 increases CD80 expression and the stimulatory capacity of bone marrow-derived dendritic cells. Int. Immunol. 10:749-755.

34. Pfeiffer, C., et al. 1995. Altered peptide ligands can control CD4 T lymphocyte differentiation in vivo. J. Exp. Med. 181:1569-1574.

35. Tao, X., Grant, C., Constant, S., and Bottomly, K. 1997. Induction of IL4-producing CD4+ T cells by antigenic peptides altered for TCR binding. J. Immunol. 158:4237-4244.

36. Chaturvedi, P., Yu, Q., Southwood, S., Sette, A., and Singh, B. 1996. Peptide analogs with different affinities for MHC alter the cytokine profile of T helper cells. Int. Immunol. 8:745-755.

37. Rogers, P.R., and Croft, M. 1999. Peptide dose, affinity, and time of differentiation can contribute to the Th1/Th2 cytokine balance. J. Immunol. 163:1205-1213.

38. Constant, S.L., and Bottomly, K. 1997. Induction of Th1 and Th2 CD4+ $\mathrm{T}$ cell responses: the alternative approaches. Annu. Rev. Immunol. 15:297-322.

39. Tan, J.T., Whitmire, J.K., Ahmed, R., Pearson, T.C., and Larsen, C.P. 1999. 4-1BB ligand, a member of the TNF family, is important for the generation of antiviral CD8 T cell responses. J. Immunol. 163:4859-4868.

40. Kuniyoshi, J.S., et al. 1999. Dentritic cell secretion of IL-15 is induced by recombinant huCD40LT and augments the stimulation of antigen-specific cytolytic T cells. Cell. Immunol. 193:48-58.

41. Kanai, T., Thomas, E.K., Yasutomi, Y., and Letvin, N.L. 1996. IL-15 stimulates the expansion of aids virus-specific CTL1. J. Immunol. 157:3681-3687

42. Young, J.W., and Steinman, R.M. 1990. Dendritic cells stimulate primary human cytolytic lymphocyte responses in the absence of CD4+ helper T cells. J. Exp. Med. 171:1315-1332.

43. Ruedl, C., Kopf, M., and Bachmann, M.F. 1999. CD8+ T cells mediate CD40-independent maturation of dendritic cells in vivo. J. Exp. Med. 189:1875-1883

44. Cella, M., Engering, A., Pinet, V., Pieters, J., and Lanzavecchia, A. 1997. Inflammatory stimuli induce accumulation of MHC class II complexes on dendritic cells. Nature. 388:782-787.

45. Lu, Z., et al. 2000. CD40-independent pathways of T cell help for priming of CD8(+) cytotoxic T lymphocytes. J. Exp. Med. 191:541-550.

46. Whitmire, J.K., et al. 1999. CD40-CD40 ligand costimulation is required for generating antiviral CD4 $\mathrm{T}$ cell responses but is dispensable for CD8 $\mathrm{T}$ cell responses. J. Immunol. 163:3194-3201. 